\title{
A quaternion-based Attitude Estimate System Based on a Low Power Consumption Inertial Measurement Unit
}

\section{Yunkun Ning}

Shenzhen Key Laboratory Low Cost

Healthcare, Shenzhen Institutes of

Advanced Technology,

Chinese Academy of Sciences,

Shenzhen 518055, China

yk.ning@siat.ac.cn

Yingnan Ma

Beijing Research Center of Urban

System Engineering, Beijing, China

\author{
Liangju Li
}

Wuhan Research Institute of

Post and Telecommunications,

Wuhan 430074,China

fordream03@gmail.com

\section{Xing Gao2}

Beijing Research Center of Urban System Engineering, Beijing, China
Guoru Zhao

Shenzhen Key Laboratory Low Cost

Healthcare, Shenzhen Institutes of

Advanced Technology,

Chinese Academy of Sciences,

Shenzhen 518055, China

gr.zhao@siat.ac.cn

Zongzhen Jin

Beijing Research Center of Urban

System Engineering, Beijing, China

\begin{abstract}
Accurate and real-time tracking of the orientation or attitude of rigid bodies has traditional applications in robotics, aerospace, underwater vehicles, human body motion capture, etc. Towards human body motion capture, especially wearable devices, the use of a longer time has always been a challenge for several weeks or several months continuously, so a low-cost chip and a low computational cost algorithm are necessary. The paper presented a quaternion-based algorithm that integrated the sensor output with the Kalman filtering algorithm, and a low power consumption Inertial Measurement Unit (IMU) for the attitude estimation. The low power consumption IMU with an inner Digital Motion Processor(DMP) from InvenSense Inc. called MPU9150, which contains triaxial accelerometers, triaxial gyroscopes, triaxial magnetometers and inner DMP. Firstly, we got attitude quaternion from DMP, and used the factored quaternion algorithm (FQA) to calculate course angle quaternion component. Then the Kalman Filtering algorithm was used to mix them together to acquire the accurate and good real-time performance attitude .The experimental results showed that Kalman filtering algorithm to mix DMP output and magnetometers data have better performance than gradient descent algorithm and complementary filter algorithm even in static performance and dynamic performance and power consumption.
\end{abstract}

\section{Categories and Subject Descriptors}

D.3.3 [Programming Languages]: Language Constructs and Features - abstract data types, polymorphism, control structures. This is just an example, please use the correct category and subject descriptors for your submission. The ACM Computing Classification Scheme: http://www.acm.org/class/1998/

\section{General Terms}

Your general terms must be any of the following 16 designated terms: Algorithms, Management, Measurement, Documentation, Performance, Design, Economics, Reliability, Experimentation, Security, Human Factors, Standardization, Languages, Theory, Legal Aspects, Verification.

\section{Keywords}

Attitude Estimate ; Kalman Filter ; Digital Motion Processor ; MPU9150 ; quaternion-based

\section{INTRODUCTION}

Accurate real-time tracking of the orientation or attitude of rigid bodies has traditional applications in robotics, aerospace, underwater vehicles, human body motion capture, etc .For human body motion capture applications, the human body can be viewed as an articulated rigid body consisting of 15 links[1]. If the orientation relative to a fixed reference frame can be determined by each link, then the overall posture of the human can accurately be rendered in real time .The attitude of a static or slow-moving individual limb segment can be measured through the attachment of an IMU module. Such IMU modules typically contain a triad of orthogonally mounted accelerometers, a triad of orthogonally mounted gyroscope and a triad of orthogonally mounted magnetometers . The gyroscope is used to measure the angular rate and its real-time performance is very good, but its performance is highly affected by the temperature. The accelerometer is used to measure the gravity vector that is relative to the coordinate frame of the IMU module, and the magnetometers offer a similar function for the local magnetic field vector . Combining accelerometers data with magnetometers data, we can get the accurate attitude, but this method is not good enough in real-time performance[2]. So we can integrate three of them to get accurate attitude and have a good real-time performance. In this paper, we used a low power consumption MEMS chip which contains triaxial accelerometers, triaxial gyroscopes, triaxial magnetometers and inner DMP . We first get attitude quaternion from DMP , and use the factored quaternion algorithm (FQA) to calculate course angle quaternion component[3-4], then using Kalman Filtering algorithm to mix them together to acquire the accurate and good real-time performance attitude. 


\section{FUSING DMP OUTPUT AND MAGNETIC DATA WITH KALMAN FILTER}

The chip we choose is called MPU9150 which contains an inner DMP. The inner DMP uses a high performance algorithm to fuse accelerometers measure data and gyroscope measure data and output attitude quaternion which has a good real-time performance[5], but the course angle of this attitude will drift with time because this algorithm didn't count magnetometers data in. Thus we used Kalman filtering algorithm to mix DMP output and magnetometers data, so we can get the attitude data which have a good real-time and a high precision, and we can use low power consumption work mode to prolong the life time of the system.

In the first step, we extracted three components from the DMP output quaternion. The way to do this is to figure out azimuth angle $\theta$, roll angle $\varphi$ and elevation angle $\psi$ at first, and then figure out the corresponding quaternion. Let us suppose the DMP output quaternion in this moment as follow[6]

$$
{ }_{A_{n}}^{E} \boldsymbol{q}_{d}=\left[w_{d} x_{d} y_{d} z_{d}\right]^{T}
$$

So, three angles are

$$
\left\{\begin{array}{c}
\theta=\arcsin \left(2\left(w_{d} y_{d}-z_{d} x_{d}\right)\right) \\
\varphi=\arctan \frac{2\left(w_{d} x_{d}+y_{d} z_{d}\right)}{1-2\left(x_{d}^{2}+y_{d}^{2}\right)} \\
\psi=\arctan \frac{2\left(w_{d} z_{d}+x_{d} y_{d}\right)}{1-2\left(y_{d}^{2}+z_{d}^{2}\right)}
\end{array}\right.
$$

The corresponding quaternion is

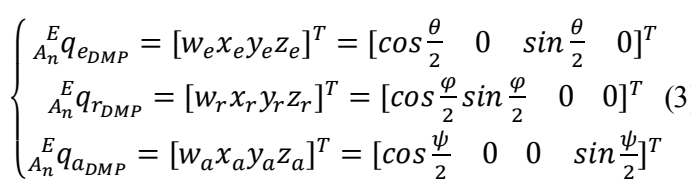

In the second step, we used this azimuth quaternion and last azimuth quaternion which we reextracted from DMP output to work out the increment quaternion ${ }_{A_{n-1}}^{A_{n}} r_{a}$

$$
{ }_{A_{n-1}}^{A_{n}} \boldsymbol{r}_{a}={ }_{A_{n-1}}^{E} \boldsymbol{q}_{a_{\mathrm{DMP}}}^{-1} *{ }_{A_{n}}^{E} \boldsymbol{q}_{a_{\mathrm{DMP}}}
$$

Then, add this increment quaternion to the azimuth quaternion that we estimated last time to get the first azimuth quaternion which we will use to do Kalman filtering[7].

$$
\boldsymbol{q}_{a_{\mathrm{DMP}}}={ }_{A_{n-1}}^{E} \boldsymbol{q}_{a} *{ }_{A_{n-1}}^{A_{n}} \boldsymbol{r}_{a}
$$

${ }_{A_{n-1}}^{\mathrm{E}} \mathrm{q}_{\mathrm{a}}$ represents the optimal estimation of azimuth quaternion last time.

In the third step, we used the FQA algorithm[8]which was developed by Xiaoping Yun in 2008 to work out the second azimuth quaternion which we will use to do Kalman filtering. as shown in the following steps.

At first, we use the current roll quaternion and elevation quaternion to rotate the measurements vector of magnetic fields ${ }^{A} m=\left[{ }^{A} m_{x}{ }^{A} m_{y}{ }^{A} m_{z}\right]^{T}$ to the earth-based coordinate system[9]

$$
{ }^{E} m={ }_{A_{n}}^{E} \boldsymbol{q}_{e_{D M P}} *{ }_{A_{n}}^{E} \boldsymbol{q}_{r_{D M P}} *{ }^{A} m *{ }_{A_{n}}^{E} \boldsymbol{q}_{a_{D M P}}^{-1} *{ }_{A_{n}}^{E} \boldsymbol{q}_{e_{D M P}}^{-1}
$$

In this expression, ${ }^{A} \mathrm{~m}$ stands for the pure vector quaternion of the 3-D vector, i.e. ${ }^{A} m=\left[\begin{array}{lll}0 & { }^{A} m_{x}{ }^{A} m_{y}{ }^{A} m_{z}\end{array}\right]^{T}$.

In the absence of measurement error, ${ }^{\mathrm{E}} \mathrm{m}$ should agree with the known local normalized magnetic field vector $\mathrm{n}=\left[\mathrm{n}_{\mathrm{x}} \mathrm{n}_{\mathrm{y}} \mathrm{n}_{\mathrm{z}}\right]^{\mathrm{T}}$, except for the effects of azimuthrotation on the sensor magnetometer readings. In such a case, $\mathrm{n}_{\mathrm{z}}={ }^{\mathrm{A}} \mathrm{m}_{\mathrm{z}}$, and

$$
\left[\begin{array}{l}
n_{x} \\
n_{y}
\end{array}\right]=\left[\begin{array}{cc}
\cos \psi_{F Q A} & -\sin \psi_{F Q A} \\
\sin \psi_{F Q A} & \cos \psi_{F Q A}
\end{array}\right]\left[\begin{array}{c}
{ }^{E} m_{x} \\
{ }^{E} m_{y}
\end{array}\right]
$$

$\psi_{F Q A}$ is the azimuth angle we want to figure out[10]. Before proceeding further, we should notice that the only difference is the direction of these two 2-D vectors, but considering that the presence of magnetic interference and measurement noise they may also differ in length. To compensate for this effect, the vectors on both sides of (7) can be normalized.

$$
\begin{gathered}
N=\left[\begin{array}{l}
N_{x} \\
N_{y}
\end{array}\right]=\frac{1}{\sqrt{n_{x}^{2}+n_{y}^{2}}}\left[\begin{array}{l}
n_{x} \\
n_{y}
\end{array}\right] \\
M=\left[\begin{array}{l}
M_{x} \\
M_{y}
\end{array}\right]=\frac{1}{\sqrt{{ }^{m_{x}^{2}+{ }^{E} m_{y}^{2}}}}\left[\begin{array}{l}
{ }^{E} m_{x} \\
{ }^{E} m_{y}
\end{array}\right]
\end{gathered}
$$

With these definitions, (7) become

$$
\left[\begin{array}{c}
N_{x} \\
N_{y}
\end{array}\right]=\left[\begin{array}{cc}
\cos \psi_{F Q A} & -\sin \psi_{F Q A} \\
\sin \psi_{F Q A} & \cos \psi_{F Q A}
\end{array}\right]\left[\begin{array}{c}
M_{x} \\
M_{y}
\end{array}\right](10)
$$

$\cos \psi_{F Q A}$ and $\sin \psi_{F Q A}$ can be solved as

$$
\left[\begin{array}{c}
\cos \psi_{F Q A} \\
\sin \psi_{F Q A}
\end{array}\right]=\left[\begin{array}{cc}
M_{x} & M_{y} \\
-M_{y} & M_{x}
\end{array}\right]\left[\begin{array}{c}
N_{x} \\
N_{y}
\end{array}\right]
$$

The azimuth angle $\psi_{\mathrm{FQA}}$ is restricted to the range(- $\pi<\psi \leq$ $\pi$ ), according to the half-angle formula, we could compute the half-angle sine and cosine values for $\psi_{\mathrm{FQA}}$

$$
\begin{gathered}
\sin \frac{\psi_{F Q A}}{2}=\operatorname{sign}\left(\sin \psi_{F Q A}\right) \sqrt{\frac{1-\cos \psi_{F Q A}}{2}} \\
\cos \frac{\psi_{F Q A}}{2}=\sqrt{\frac{1+\cos \psi_{F Q A}}{2}}
\end{gathered}
$$

The azimuth quaternion is then given by

$$
\boldsymbol{q}_{a_{F Q A}}=\left[\begin{array}{llll}
\cos \frac{\psi_{F Q A}}{2} & 0 & 0 & \sin \frac{\psi_{F Q A}}{2}
\end{array}\right]^{T}
$$

Now, we get two azimuth quaternion $\mathrm{q}_{\mathrm{a}_{\mathrm{DMP}}}$ and $\mathrm{q}_{\mathrm{a}_{\mathrm{FQA}}}$ which will be used in Kalman filter, in Kalman filter application, we treat $\mathrm{q}_{\mathrm{a}_{\mathrm{DMP}}}$ as the predictions, and $\mathrm{q}_{\mathrm{a}_{\mathrm{FQA}}}$ as the measurements.

In the first step of Kalman filtering - we predict current azimuth quaternion based on the optimal estimation last time. As (5), $\underset{A_{n-1}}{E_{q}} q_{a}$ is the optimal estimation of last time, ${ }_{A_{n-1}}^{A_{n}} r_{a}$ is the predicted increment, $\mathrm{q}_{\mathrm{a}_{\text {DMP }}}$ is the predictions of this moment[11].

In the second step of Kalman filtering - update the corresponding auto-covariance. For simplifying the computation, we use algebraic sum in this paper

$$
{ }_{n-1}^{n} P={ }_{n-1}^{n-1} P+Q
$$


${ }_{n-1}^{\mathrm{n}-1} \mathrm{P}$ is the auto-covariance of last state and $\mathrm{Q}$ is the a uto-covariance of system procedures.

In the third step of Kalman filtering - combine predictions with measurements to get the optimal estimation azimuth quaternion

$$
{ }_{A_{n}}^{E} \boldsymbol{q}_{a}=\operatorname{slerp}\left(\boldsymbol{q}_{a_{D M P}}, \boldsymbol{q}_{a_{F Q A}}, K g\right)
$$

In this formula, slerp represents the interpolation calculation of quaternion, $\mathrm{q}_{\mathrm{a}_{\mathrm{DMP}}}$ is the starting point and $\mathrm{q}_{\mathrm{a}_{\mathrm{FQA}}}$ is the ending point of interpolation calculation, $\mathrm{Kg}$ is interpolation coefficient (gain of Kalman filter). The way to compute $\mathrm{Kg}$ is as following

$$
K g={ }_{n-1}^{n} P /\left({ }_{n-1}^{n} P+R\right)
$$

In the fourth step of Kalman filtering -So far we have obtained the optimal estimation of the state, but for the next step, we should update the auto-covariance of current state

$$
{ }_{n}^{n} P=(1-K g) *{ }_{n-1}^{n} P
$$

The most critical value in the process of designing Kalman filter are $\mathrm{Q}$ and $\mathrm{R}$, which determine the velocity of error correction process and attitude smooth degree.

Since we have got the optimal azimuth quaternion, we should combine the three quaternion again to get a complete attitude quaternion, methods are as follows

$$
{ }_{A_{n}}^{E} \boldsymbol{q}={ }_{A_{n}}^{E} \boldsymbol{q}_{a} *{ }_{A_{n}^{E}}^{E} \boldsymbol{q}_{e} *{ }_{A_{n}}^{E} \boldsymbol{q}_{r}
$$

$$
E(x)=\sum_{i=1}^{n} x_{i} / n
$$

Then calculation squared variance is

$$
D(x)=\sum_{i=1}^{n}\left(\bar{x}-x_{i}\right)^{2} / n
$$

We evaluate the performance by comparing the squared covariance. The result of this experiment is as follows:
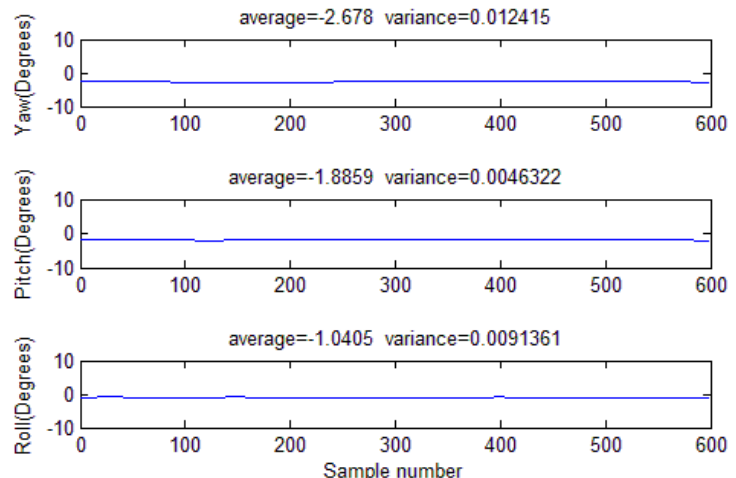

Fig. 2. Static performance of gradient descent algorithm

1) Gradient descent algorithm

Taken the $\beta=0.02$ times gradient as the step size of data fusion, because when $\beta<0.02$ the correct effect can't refrain integral floating of gyroscope.

2) Complementary filter algorithm

To simplify calculations, we take the same correct coefficient $\alpha$ and $\beta$ as 0.02 , the same as gradient descent algorithm, when $\beta<0.02$ the correct effect can't refrain integral floating of gyroscope[14].
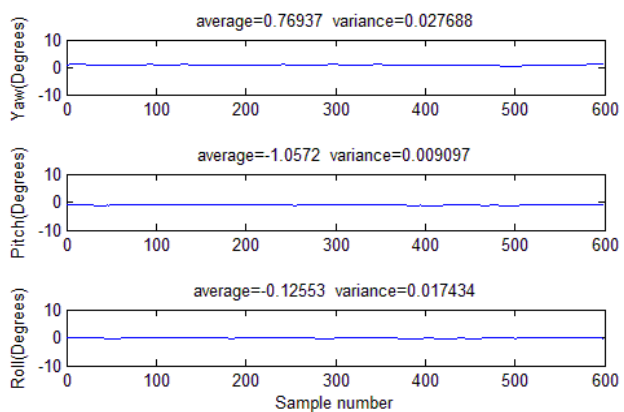

Fig. 1. The complete algorith $m$ flow of Kalman

\section{EXPERIMENTAL RESULTS}

To compare the arithmetic performance, we present two studies to compare with gradient descent algorithm [12-13] and complementary filter algorithm in static and dynamic performance.

\section{A. Static performance experiment}

In this experiment, we place the sensor module static for 20 seconds at first, and then the sensor samples the inertia data continuously for 12 seconds, the sampling frequency is $50 \mathrm{~Hz}$.

The method for data processing is to calculate the mean value of output value in each axis
Fig. 3. Static performance of complementary filter algorithm

3) Kalman filtering algorithm to mix DMP output and magnetometers data

The core of this algorithm is to determine the auto covarianceQ of system and auto covariance $\mathrm{R}$ of measurements in Kalman filtering. We do not care the pitch angle and roll angle because these two angles are from DMP directly.

We list only two cases in graphics, and others will be archived as tables.

(a) $Q=0.0001, R=0.9$ 


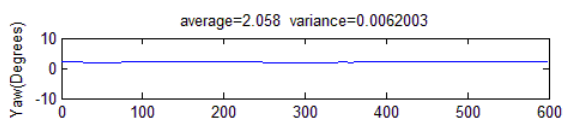

(क average $=-1.3499$ variance $=3.6323 \mathrm{e}-005$
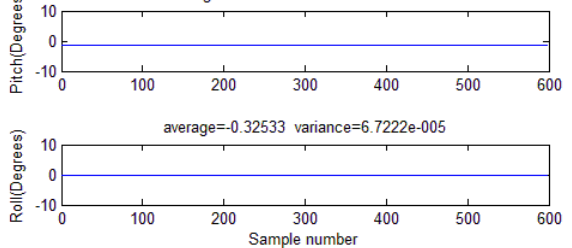

(b) $\mathrm{Q}=0.0001, \mathrm{R}=0.9$
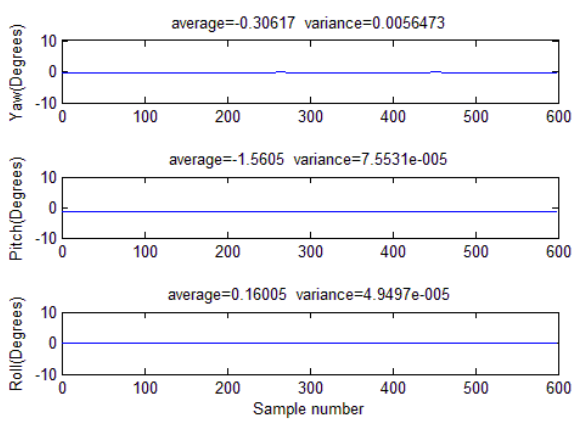

Fig. 4. Static performance of Kalman filtering algorithm Other cases are as follows

TABLE 1. Yaw angles variances in Kalman filtering algorithm

\begin{tabular}{|l|r|r|r|r|}
\hline $\mathrm{R}$ & 0.9 & 0.5 & 0.2 & 0.1 \\
\hline 0.001 & 0.033488 & 0.032642 & 0.051927 & 0.077798 \\
\hline 0.0001 & 0.0056473 & 0.009667 & 0.018256 & 0.03065 \\
\hline 0.00001 & 0.0010411 & 0.0016374 & 0.0027799 & 0.0062003 \\
\hline
\end{tabular}

Show as figue 5 is below

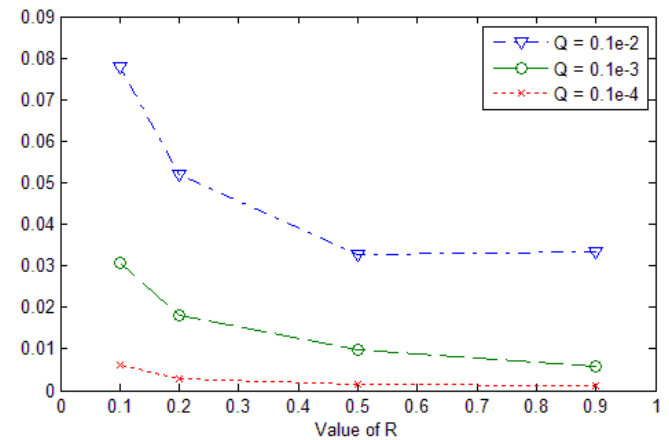

Fig. 5. Yaw angles variance strends

\section{B. Dynamic performance experiment}

In this experiment, we make sure that it is done under the static condition and in order to test the dynamic performance of each algorithm, the sampling rate is $50 \mathrm{~Hz}$. then the pitch angle increases from $0^{\circ}$ to $90^{\circ}$ and then decreases from $90^{\circ}$ to $0^{\circ}$.

From above section, we choice the $\beta=0.02$ times gradient as the step size of gradient descent algorithm, 0.02 as the correct coefficient of complementary filter algorithm and $Q=$ $0.00001, R=0.1$ as the coefficients of Kalman filtering algorithm to observe the dynamic performance, results are as follows[15-16]
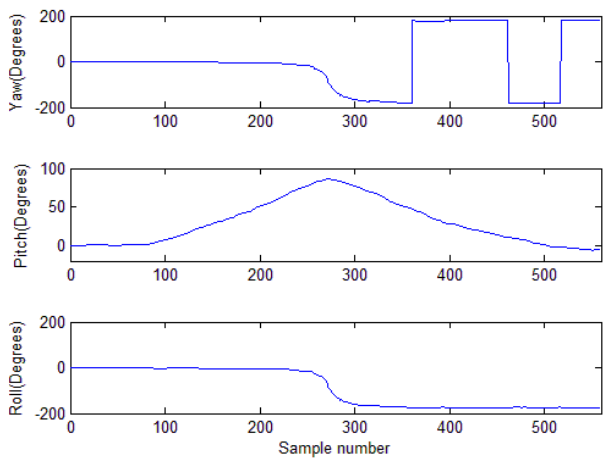

Fig. 6. Dynamic performance of gradient descent algorithm $\beta=0.02$
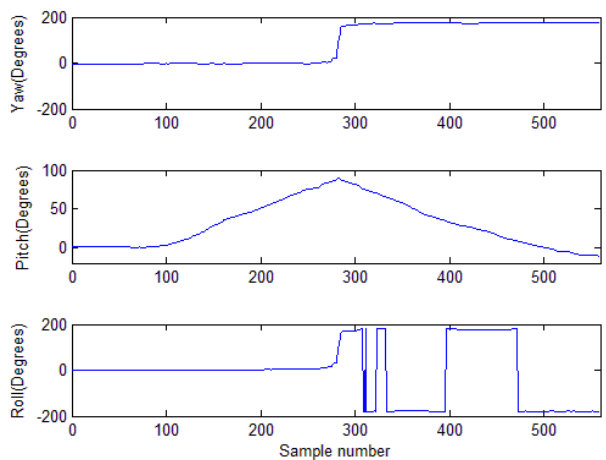

Fig. 7. Dynamic performance of complementary

Algorithm $\alpha=\beta=0.02$
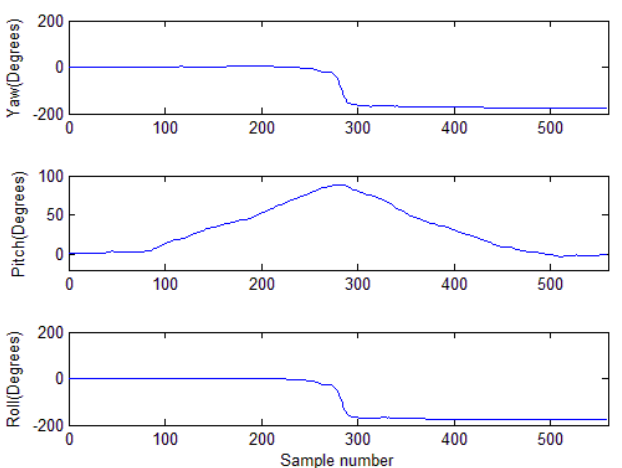

Fig. 8. Dynamic performance of Kalman filtering algorithm $Q=0.00001, R=0.1$ 
To observe dynamic performance more directly, we design another experiment to compare the dynamic performance of these three algorithm -- fast-spinning the yaw angle from $0^{\circ}$ to $180^{\circ}$, and then we observe the result.
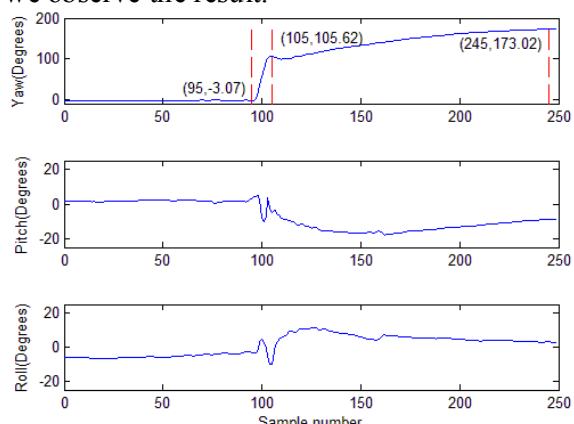

Fig. 9. Fast spinning performance of gradient descent algorithm
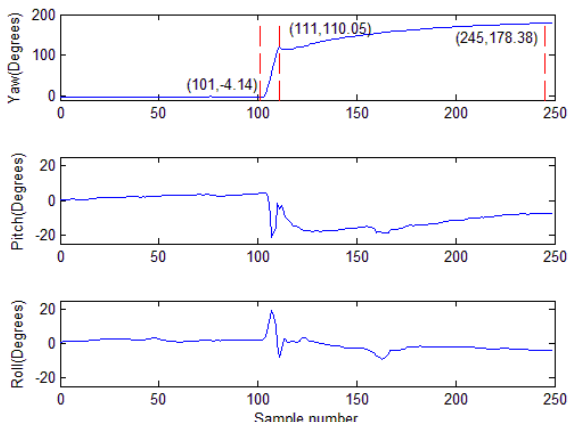

Fig. 10. Fast spinning performance of complementary filter algorithm
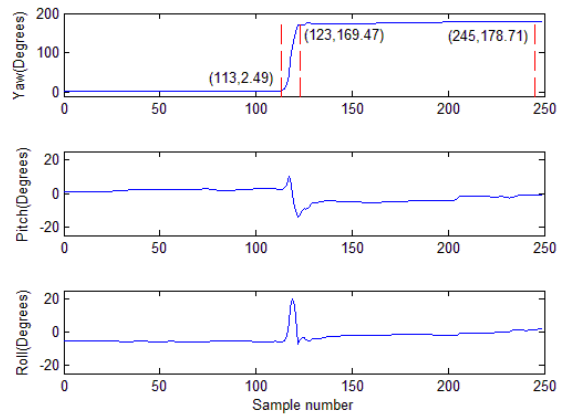

Fig. 11. Fast spinning performance of Kalman filtering algorithm

\section{CONCLUSION}

We can draw conclusions from two experiment results above - Kalman filtering algorithm to mix DMP output and magnetometers data have better performance than gradient descent algorithm and complementary filter algorithm even in static performance and dynamic performance.

This paper presents a quaternion-based attitude estimate system based on a low cost inertial measurement unit. The Kalman filter produces estimates in quaternion form through a series of sequential rotations. These rotations can individually be examined in a manner similar to azimuth angle $\theta$, roll angle $\varphi$ and elevation angle $\psi$ in a Euler angle sequence. However, unlike Euler angles ,the algorithm incorporates a singularity avoidance method. In the algorithm, magnetometer data are not used to calculate orientation that is relative to the vertical axis; thus, magnetic variations result in errors only in the horizontal plane.

This property of the system is experimentally demonstrated . Singularities in the numerical implementation are avoided through the use of a method that assigns an offset body coordinate system when a singularity occurs. The algorithm is efficient and does not require the evaluation of trigonometric functions. Experimental results indicate that, when combined with a low-pass filter for accelerometer data, the algorithm is able to track orientation. The system has been successfully used in real-time human body motion tracking applications.

\section{ACKNOWLEDGMENT}

This study has been financed partially by the National Natural Science Foundation of China (Grant No. 51105359), the National Key Technology R\&D Program (Grant No. 2015BAI06B02) and the National 863 Programs of China (Grant No. 2012AA02 A604), and was also supported by the Shenzhen science and technology research and development fund(No.78922035X-JCYJ20130401170412293) and Beijing Financial Fund (Grant No. PXM2015_178215_000002).

\section{REFERENCES}

[1] Madgwick S O H, Harrison A J L, Vaidyanathan R. Estimation of IMU and MARG orientation using a gradient descent algorithm[C]/Rehabilitation Robotics (ICORR), 2011 IEEE International Conference on. IEEE, 2011: 1-7.

[2] Euston M, Coote P, Mahony R, et al. A complementary filter for attitude estimation of a fixed-wing UAV[C]//Intelligent Robots and Systems, 2008. IROS 2008. IEEE/RSJ International Conference on. IEEE, 2008: 340-345.

[3] R. A. Hyde, L. P. Ketteringham, S. A. Neild, and R. J. S. Jones, "Estimation of upper-limb orientation based on accelerometer and gyroscope measurements," vol. 55, pp. 746-754, Feb. 2008.

[4] J. F. Vasconcelos, G. Elkaim, C. Silvestre, P. Oliveira, and B. Cardeira,"A geometric approach to strapdown magnetometer calibration in sensor frame," in Navigation, Guidance and Control of Underwater Vehicles,vol. 2, 2008.

[5] Xsens Technologies B.V., MTi and MTx User Manual and Technical Documentation. Pantheon 6a, 7521 PR Enschede, The Netherlands, May 2009.

[6] MicroStrain Inc., 3DM-GX3 -25 Miniature Attutude Heading Reference Sensor. 459 Hurricane Lane, Suite 102, Williston, VT 05495 USA, 1.04 ed., 2009.

[7] VectorNav Technologies, LLC, VN -100 User Manual. College Station,TX 77840 USA, preliminary ed., 2009.

[8] InterSense, Inc., InertiaCube2+ Manual. 36 Crosby Drive, Suite 150,Bedford, MA 01730, USA, 1.0 ed., 2008.

[9] Vicon Motion Systems Limited., Vicon Nexus Product Guide - Foundation Notes. 5419 McConnell Avenue, Los Angeles, CA 90066, USA,1.2 ed., November 2007.

[10] P. Martin and E. Salan, "Design and implementation of a low-costobserver-based attitude and heading reference system," Control Engineering Practice, vol. 18, no. 7, pp. 712 - 722, 2010. Special Issue on Aerial Robotics.

[11] X. Yun, E. R. Bachmann, H. Moore, and J. Calusdian, "Self-contained position tracking of human movement using small inertial/magnetic sensor modules," in ICRA, 
pp. 2526-2533, 2007.

[12] H. M. Schepers, H. Koopman, and P. H. Veltink, "Ambulatory assessment of ankle and foot dynamics," IEEE Transactions on Biomedical Engineering, vol. 54, no. 5, pp. 895-902, 2007.

[13] R. Stirling, K. Fyfe, and G. Lachapelle, "Evaluation of a new method of heading estimation for pedestrian dead reckoning using shoe mounted sensors," The Journal of Navigation, vol. 58, no. 01, pp. 31-45, 2005.

[14] F. Cavallo, A. Sabatini, and V. Genovese, "A step toward gps/ins personal navigation systems: real-time assessment of gait by foot inertial sensing,"pp. 1187 - 1191, aug. 2005.

[15] G.Zhao, Z.Mei, D. Liang, K.Ivanov, Y.Guo, Y.Wang and L.Wang. Exploration and Implementation of a Pre-Impact Fall Recognition Method Based on an Inertial Body Sensor Network, Sensors. 12 (11), 2012, 15338-15355.

[16] D.Liang, G.Zhao, Y.Guo and L.Wang. Pre-impact \& Impact Detectionof Falls Using Wireless Body Sensor Network. IEEE-EMBS International Conference on Biomedical and Health Informatics, Shenzhen, China, January 2-7, 2012: 763-766 
